TRABAJOS DE PREHISTORIA

53, n. $^{\circ} 1,1996$, pp. $147-154$

\section{HALLAZGO DE LOS PRIMEROS UTENSILIOS DE MADERA EN EL POBLADO NEOLÍTICO DE LA DRAGA (BANYOLES, GIRONA)}

THE FINDING OF THE FIRST WOODEN TOOLS IN THE NEOLITHIC SITE OF LA DRAGA (BANYOLES, GIRONA)

ÁNGEL BOSCH LLORET (*)

JULIA CHINCHILLA SÁNCHEZ (*)

RAQUEL PIQUÉ HUERTA (**)

JOSEP TARRÚS GALTER (*)

\section{RESUMEN}

La Draga es un poblado neolítico de finales del VI milenio que se encuentra en la orilla oriental del lago de Banyoles. El resultado más destacable de la excavación en el sector subacuático ha sido la aparición de cuatro utensilios de madera, objetos hasta ahora poco conocidos en la prehistoria de la zona mediterránea.

\begin{abstract}
La Draga is a neolithic site of the later 6th millennium located in the eastern border of the Banyoles lake. The most important result of the excavation in the sub aquatic zone has been the finding of four wooden tools, a kind of object rarely known in the Mediterranean Prehistory till now.

(*) Directores del Proyecto de excavación. Museu Arqueològic Comarcal de Banyoles. Plaça de la Font, 11. 17820. Banyoles.

(*) Laboratori d'Anàlisis Arqueològiques. Departament de Prehistòria. Universitat Autònoma de Barcelona. 08103. Bellaterra.

El artículo fue remitido en su versión final el 22-III-96.
\end{abstract}

Palabras clave: Neolítico antiguo. Poblado lacustre. Excavación subacuática. Utensilios de madera. Nordeste de Cataluña.

Key words: Early Neolithic. Lacustrian site. Sub aquatic excavation. Wooden tools. North-east of Catalonia.

\section{INTRODUCCIÓN}

El hallazgo de objetos prehistóricos de madera u otras fibras vegetales es un fenómeno excepcional limitado a condiciones particulares de conservación, ya sea de permanente humedad o de extrema aridez. En Europa, este tipo de hallazgos han sido frecuentes en medios húmedos (lagos y turberas) de las zonas continentales y atlánticas, en las que se ha obtenido un importante conjunto de útiles cuyas interpretaciones funcionales, basándose en afinidades geográficas o cronoculturales, se han adoptado en las correspondientes a los utensilios líticos y óseos de las 
zonas mediterráneas, aun a sabiendas de que los condicionantes culturales y medioambientales de cada zona podían haber aportado elementos característicos y diferenciadores propios.

Sin embargo, en el Mediterráneo occidental se conocen hallazgos puntuales en medios áridos, como los de la cueva de los Murciélagos (Góngora, 1868) o la cueva Sagrada de Lorca (Ayala, 1987, 1990), que en los últimos años se han visto considerablemente enriquecidos por los realizados en los primeros asentamientos lacustres neolíticos descubiertos: La Marmotta, en un pequeño lago en el centro de la península italiana (Fugazzola et alii, 1993) y La Draga, a orillas del lago de Banyoles (Girona), en el Nordeste de la Península Ibérica.

El yacimiento arqueológico de La Draga fue descubierto en abril de 1990 en la orilla oriental del lago de Banyoles, conocido localmente como l'estany, como consecuencia de las obras de construcción de un parque en la sede olímpica de remo de los juegos olímpicos de 1992. Se trata de un poblado al aire libre de finales del VI milenio, dentro de la tradición de cerámicas impresas de la orilla occidental del Mediterráneo. Su situación en una zona pantanosa, y periódicamente inundada, ha proporcionado una conservación extraordinaria de los restos orgánicos (Fig. 1).

En mayo de 1990 se realizó una primera campaña de prospección para delimitar el asentamiento y, a partir de los buenos resultados obtenidos, se inició al año siguiente la excavación arqueológica sistemática en una zona situada a unos 50 metros de la orilla. Ésta y las siguientes campañas de 1992 y 1994 han permitido documentar (Tarrús et alii, 1992, 1993; 1994a, b) un gran número de estructuras construidas directamente sobre el suelo de creta lacustre, entre las que destacan las de combustión (hogares) y las plataformas enlosadas de travertino.

Sin embargo, la principal característica de este yacimiento es la multitud de agujeros de poste identificados que conservan en su interior los restos de las puntas de madera, siempre a partir de unos $60 \mathrm{~cm}$. por debajo del nivel arqueológico, debido a las condiciones freáticas de la zona. Estos fragmentos de pilares de madera, de los que el mayor conservado llega a medir más de dos metros de largo, permitirán

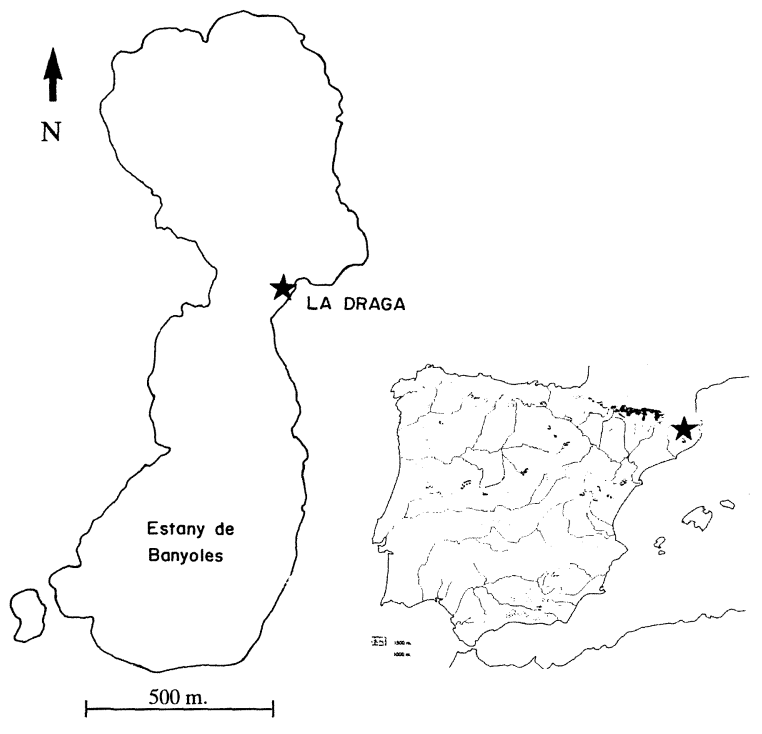

Fig. 1. Situación del poblado neolítico de La Draga en la orilla oriental del lago de Banyoles (Girona).

reconstruir, después de su análisis dendrocronológico, la forma de las estructuras y su cronología relativa en relación al período de ocupación del poblado.

La determinante edafológica de la zona excavada es, sin duda, la oscilación permanente del nivel de la capa freática, ocasionalmente superior a 1 metro en un mismo año. Ésta ha provocado que los restos de madera sólo puedan conservarse a partir del nivel mínimo de oscilación. Pero, por otra parte, la vegetación adaptada a estas condiciones creó un suelo de turba después del abandono del poblado que, aunque no permitió la conservación de la madera, sí lo hizo de otros restos orgánicos.

De esta forma, los frutos, semillas y restos de maderas carbonizadas, y los restos de fauna pueden contabilizarse por miles, aportando una valiosa información paleoeconómica sobre sus antiguos pobladores. De forma parecida puede calificarse la conservación de los restos de la cultura material, excepto en el caso de la cerámica, que es el elemento que peor ha soportado la oscilación continua del grado de humedad. Aun no siendo el objeto de este artículo, no quisiéramos dejar de citar algunos elementos singulares dentro de la cultura material, como es el caso de varios fragmentos de un pequeño vaso de mármol y un resto de talla

T. P., 53, n. ${ }^{\circ} 1,1996$ 
de obsidiana. Elementos que pueden indicar una relación con tierras lejanas del Mediterráneo central.

La excepcionalidad de La Draga guardaba aún una nueva sorpresa cuando en el año 1994, dentro de los trabajos de delimitación del poblado, se solicitó la intervención del Centre d'Arqueologia Subaquàtica de Catalunya, para prospectar el interior del lago, en la orilla situada frente al yacimiento. El objetivo de esta intervención era el de determinar la situación de esta orilla durante el período de ocupación.

Los primeros resultados pusieron de manifiesto que el asentamiento neolítico continúa unos metros en el interior del lago y que el suelo arqueológico se encuentra a 2,05 m. por debajo del nivel medio actual del agua y a 1,45 $\mathrm{m}$. del fondo lacustre, en el punto más cercano a la orilla. De esta forma, se han incrementado enormemente las posibilidades arqueológicas de la excavación, así como la complejidad de los trabajos a realizar. A partir de 1995 este organismo se ha incorporado a la excavación arqueológica, que ahora se realiza en dos sectores diferentes, uno terrestre y otro subacuático, con metodologías adaptadas a las condiciones medioambientales de cada caso, que se complementan entre sí.

Con esta intervención, el Centre d'Arqueologia Subaquàtica de Catalunya, dirigido por Xavier Nieto, realiza su primera excavación en un período prehistórico y en un medio de aguas interiores. Fruto destacado de ello es el hallazgo de los cuatro utensilios de madera que presentamos.

\section{PRIMEROS UTENSILIOS DE MADERA}

Los cuatro objetos hallados en la Draga se encuentran actualmente en proceso de análisis y de restauración (secado por liofilización) en el Museo cantonal de Neuchâtel (Suiza); sin embargo, el interés que tienen para el conocimiento del Neolítico peninsular justifica su presentación, aunque limitada por el momento a una breve descripción de sus características y a un intento de determinar su probable funcionalidad.
El primer utensilio (Lám. I) es de madera de roble (1). Mide $29 \mathrm{~cm}$. de largo por $14,5 \mathrm{~cm}$. de ancho. Presenta un mango cilíndrico de $20 \mathrm{~cm}$. de largo y $2 \mathrm{~cm}$. de diámetro, finalizado en el extremo proximal por una protuberancia subesférica. La parte activa del objeto, que se encuentra en el extremo distal, recuerda a la hoja de una hoz y, como ella, finaliza con un extremo apuntado y presenta un filo cortante en un perfil cóncavo de $11 \mathrm{~cm}$. Por el contrario, el perfil opuesto es completamente recto y de sección roma.

En el perfil activo han quedado marcadas numerosas señales de utilización, que sugieren una mecánica de uso parecida a la empleada con una hoz, probablemente sobre fibras vegetales no leñosas. La robustez que presenta el filo cortante y la ausencia de aplicación de un elemento auxiliar como el sílex podría sugerir una función destinada más a facilitar una labor de arrancar los tallos, que no la de cortarlos propiamente, dentro de una supuesta actividad de tipo recolector. Desconocemos, por el momento, cualquier paralelo dentro del registro arqueológico europeo.

El segundo utensilio (Lám. II) es de madera de saúco y mide $20,2 \mathrm{~cm}$. de longitud por $14 \mathrm{~cm}$. de anchura. Se trata básicamente de un soporte cilíndrico de $2,2 \mathrm{~cm}$. de sección media, en el que se halla la zona de enmangamiento, finalizada igualmente en el extremo proximal por̈ una protuberancia subesférica, y la zona activa. En esta última, a $11 \mathrm{~cm}$. del extremo proximal, se ha practicado una ranura romboidal en la cual se ha encastado una lámina de sílex, de la que sólo se conserva la mitad proximal. La lámina se encuentra pegada mediante una resina (no determinada aún) y, en sección transversal, está orientada en relación al soporte en el mismo eje direccional, mientras que, en sección longitudinal lo está de forma oblicua, formando un ángulo inferior a $45^{\circ}$.

En el lado anverso, el soporte cilíndrico presenta una profunda ranura longitudinal, de 6 a $9 \mathrm{~mm}$. de anchura por $6 \mathrm{~mm}$. de profundidad, desde el interior de la cual puede observarse el extremo proximal de la lámina de sílex. Final-

(1) Para la identificación de la madera se ha partido de los criterios anatómicos proporcionados por Schweingruber (1978; 1990) y Carlquist (1988), utilizando como material comparativo la colección de referencia de maderas actuales del Servei d'Anàlisi Arqueològiques de la Universitat Autònoma de Barcelona.

T. P., 53, n. ${ }^{\circ} 1,1996$ 

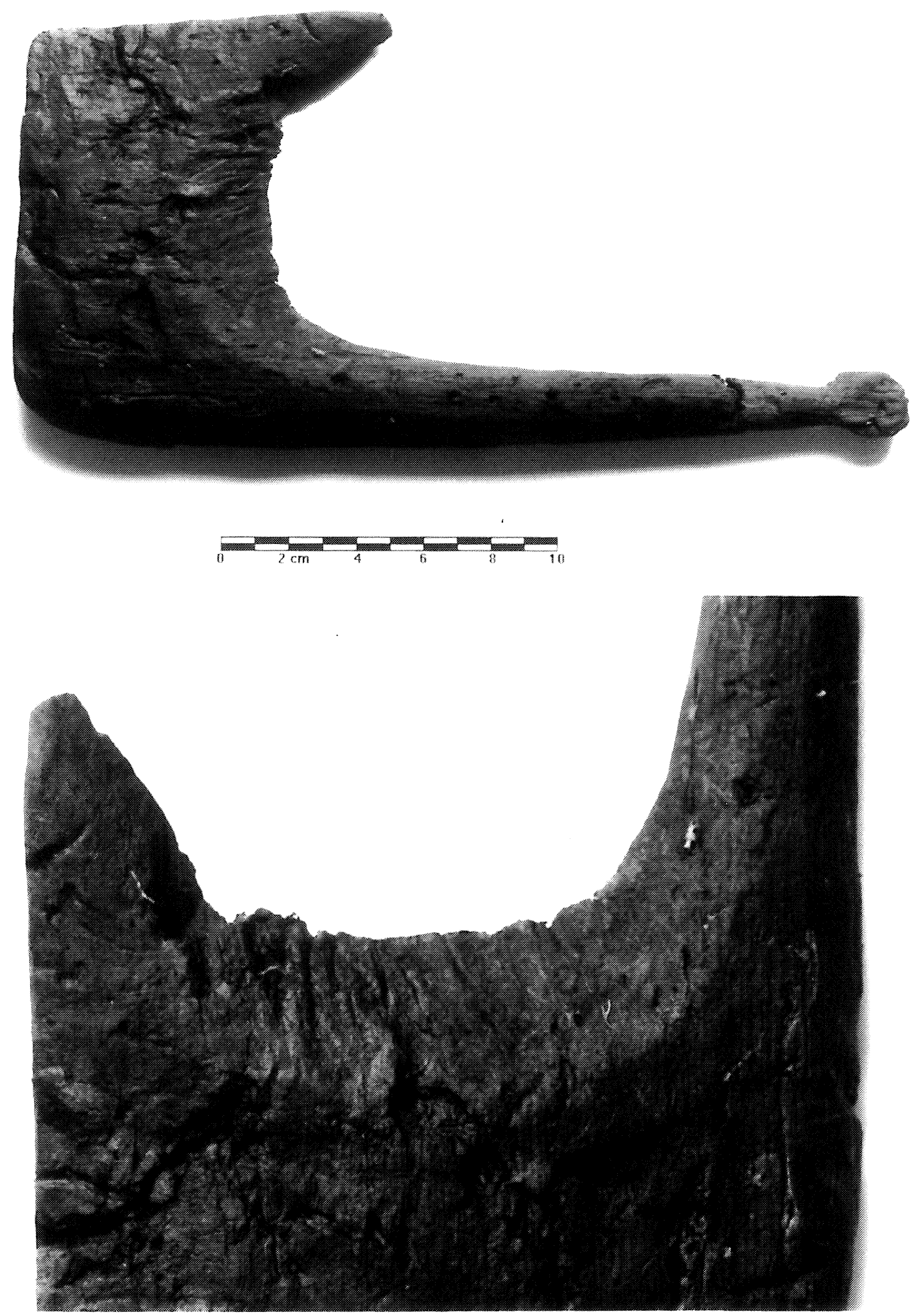

Lám. I. Utensilio de madera número 1 y detalle del filo cortante con señales de uso (poblado neolítico de La Draga, Banyoles, Girona).

T. P., 53, n. ${ }^{\circ} 1,1996$ 


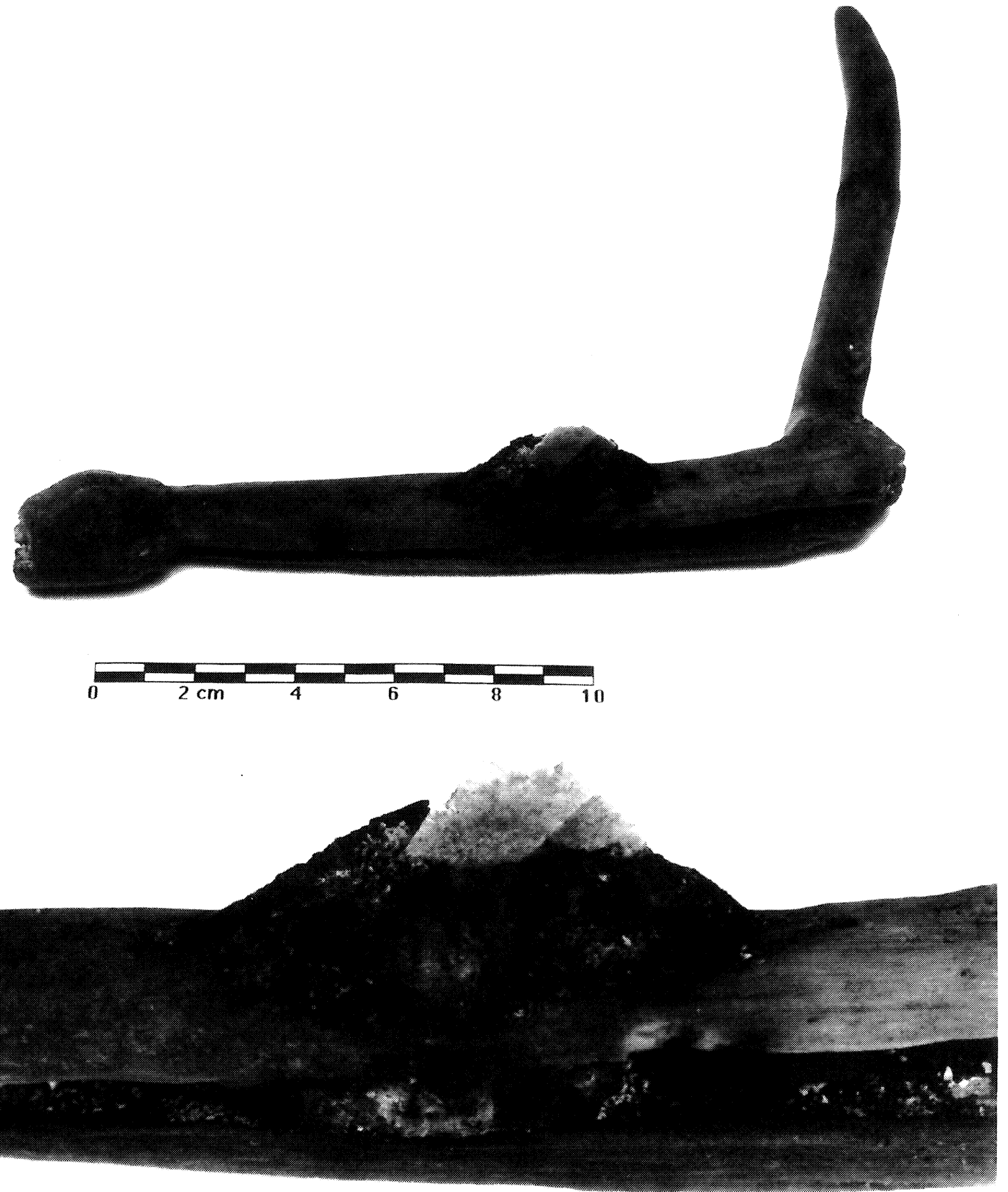

Lám. 2. Utensilio de madera número 2 y detalle de la sujeción de la hoja de sílex mediante resina (poblado neolítico de La Draga, Banyoles, Girona).

mente, en el extremo distal, existe una protuberancia curva de $12 \mathrm{~cm}$. de longitud, de sección circular, de $1,3 \mathrm{~cm}$. de diámetro, realizada a partir de una rama de la propia planta. Se sitúa

T. P., 53, n. ${ }^{\circ} 1,1996$ 

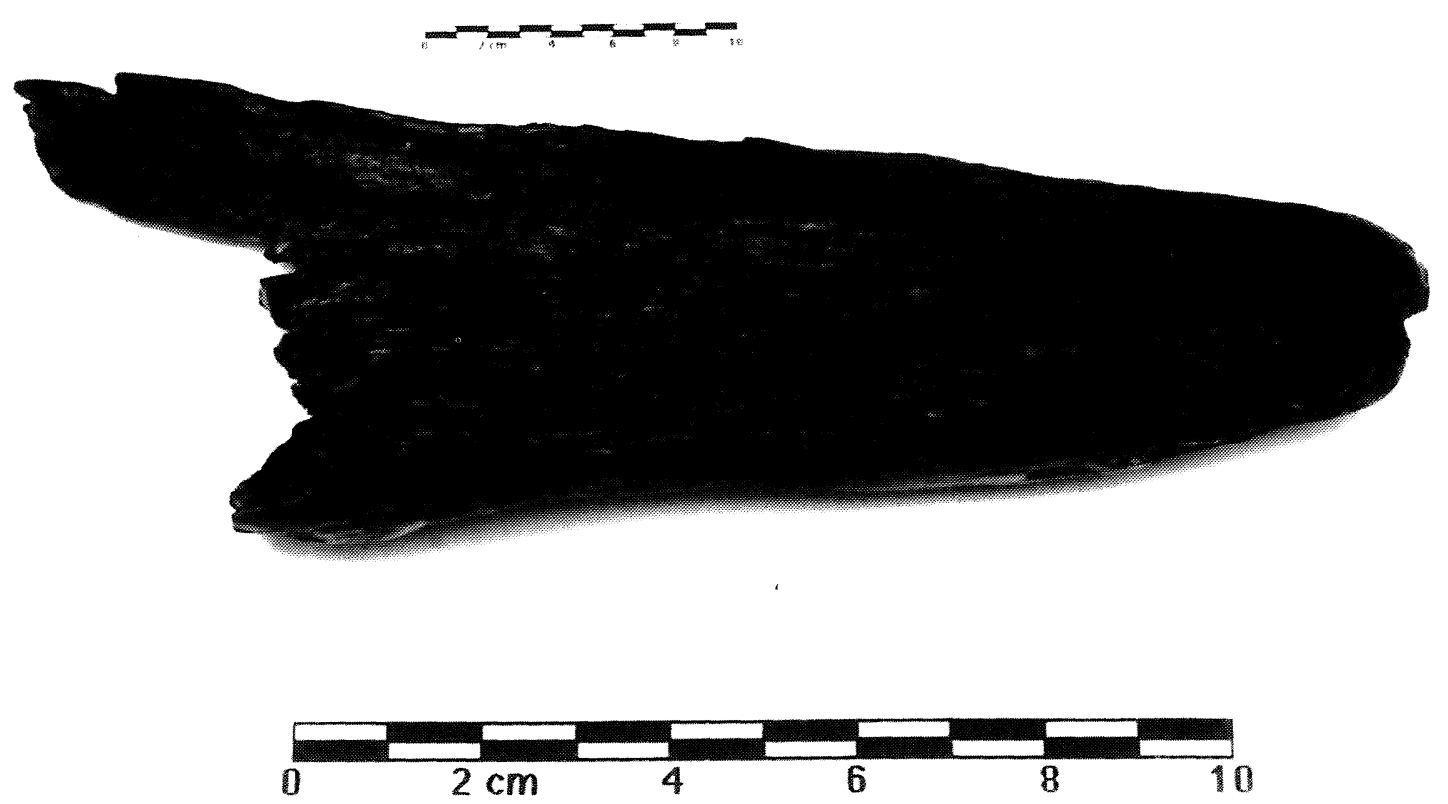

Lám. III. Utensilios de madera números 3 y 4 del poblado neolítico de La Draga (Banyoles, Girona).

transversalmente al eje de enmangue y a un plano diferente, tanto de la hoja de sílex como de la ranura longitudinal.

Es un utensilio que presenta numerosas afinidades con los descubiertos en las fases medias (Cortaillod y Pfyn antiguos) del Neolítico de la Región Alpina, donde han sido clasificados como hoces con enmangamiento oblicuo directo, tal como aparecen por ejemplo en Egolzwil LU (Baudais, 1989; Mueller-Beck, 1965; Voruz, 1991; Schlichtherle, 1992).

$\mathrm{Su}$ uso, en principio, parece de fácil concepción: un extremo distal para recoger los tallos que son apresados por la mano libre, para seguidamente dar una pequeña rotación al mango y situar a la hoja de sílex en el plano de corte. Sin embargo, resulta difícil evidenciar la utilidad de la ranura longitudinal (¿posible reutilización de un mango que llevaba láminas de sílex encastadas en paralelo?, ¿adición de algún elemento auxiliar?, ¿facilitar el recambio de la hoja presionando desde el interior de la ranura?, etc.).

T. P., 53, n. ${ }^{\circ} 1,1996$
El tercer utensilio (Lám. III) está realizado con madera de acebuche y mide $52,4 \mathrm{~cm}$ de longitud por $2,1 \mathrm{~cm}$ de diámetro máximo. Se trata de un bastón recto de forma cilíndrica, que presenta una superficie completamente regularizada y pulimentada. El extremo proximal es completamente romo y no presenta señales de haber sido zona activa. En las proximidades de este extremo aparecen varias incisiones transversales, paralelas entre sí. Por contra, el extremo proximal está fracturado.

Probablemente se trata de un bastón o vara, objeto de uso personal, destinado a numerosos usos no específicos.

El cuarto utensilio (Lám. III) es de madera de roble. Se encuentra afectado por la combustión y se conserva sólo parcialmente. Presenta una forma cónica, con el interior ahuecado, con una longitud de $17,5 \mathrm{~cm}$. y una sección máxima de $5,5 \mathrm{~cm}$. Posiblemente, se trate de una vaina o funda de un objeto lítico u óseo, que formaría parte de un utensilio complejo. 
Finalmente, podrían hacerse algunas apreciaciones sobre la elección de la madera empleada:

- El roble (Quercus sp.) es la especie más explotada en La Draga sobre todo como elemento constructivo. En la confección de útiles su madera, dura y compacta, ha sido seleccionada en los utensilios primero y cuarto, que requerían como elemento común una gran resistencia.

- La opción por el saúco (Sambucus sp.), en el segundo caso, parece que está motivada por la facilidad de realizar ranuras aprovechando la médula blanda del interior de sus ramas, hecho que se ha podido constatar por experimentación (2). Por otra parte, es una especie bien adaptada a las condiciones ambientales de humedad y que debió ser frecuente en las inmediaciones del poblado.

- Finalmente, la opción del acebuche (Olea europaea) debió estar determinada por el hecho de ser ésta una de las maderas más compactas y homogéneas y con posibilidades de adquirir un buen pulimento, motivos por los que aún actualmente es muy apreciada en diferentes artes que trabajan la madera, siendo utilizada en la fabricación de bastones, también, por su gran rigidez. Sin embargo, es una especie de clima mediterráneo seco, que no concuerda con la vegetación potencial que aportan los análisis paleoambientales. Se la podría considerar como un aporte foráneo. Actualmente, el acebuche se encuentra en la costa ampurdanesa, a unos 50 kilómetros del yacimiento.

\section{CONCLUSIONES}

La excavación de un yacimiento sobre un medio permanentemente húmedo, como es el caso de La Draga, precisa de una infraestructura mucho más compleja, pero, a cambio, proporciona una cantidad y calidad de información que difícilmente puede darse en los yacimientos sobre medio seco.

(2) De forma complementaria al análisis de estos objetos de madera, el prehistoriador Antoni Palomo Pérez, participante en el proyecto de excavación y con una ya larga trayectoria en la experimentación de utensilios prehistóricos, ha reproducido de forma sistemática todo el proceso de manufactura de estos útiles con un doble objetivo: analítico y didáctico.
La conservación de objetos de madera aporta una visión mucho más completa del instrumental utilizado por las poblaciones neolíticas, a la vez que sirve como punto de referencia para constatar la funcionalidad de los numerosos objetos líticos y óseos proporcionados por las excavaciones. Por otra parte, es un nuevo elemento a considerar en la explotación del medio ambiente circundante.

La continuación de la excavación en la zona subacuática de La Draga (Banyoles) debe aportar nuevos utensilios de madera que nos ayudarán a comprender mejor una parte esencial de la cultura material del Neolítico antiguo mediterráneo, hasta ahora muy poco conocida.

\section{BIBLIOGRAFÍA}

AyalA, M. ${ }^{a}$ M. (1987): "Enterramientos calcolíticos en la Sierra de Tercia, Lorca. Murcia. Estudio preliminar". Anales de Prehistoria y Arqueología, 3: 9-24. Universidad de Murcia.

- (1990): "Estudio preliminar del ritual funerario calcolítico en la comarca de Lorca, Murcia". Zephyrus, XLIII: $77-86$.

BAUDAIS, D. (1989): "Le mobilier en bois et en écorce du niveau V'. En P. Petrequin (ed.): Les sites littoraux néolithiques de Clairvaux-Les-Lacs (Jura), 2: le néolithique moyen. Paris: 349-362.

BoscH, A. (1994): "El Neolítico antiguo en el Nordeste de Cataluña. Contribución a la problemática de la evolución de las primeras comunidades neolíticas en el Mediterráneo occidental". Trabajos de Prehistoria, 51, 1: 55-75. Madrid.

CARlquist, S. (1988): Comparative wood anatomy. Springer-Verlag, Berlin.

Fugazzola, M.A. ; D'Eugenio, G. y Pessina. A. (1993): "La Marmotta (Anguillara Sabazia, R.M.). Scavi 1989. Un abitato perilacustre di età neolitica". Bulletino di paletnologia italiana, 84, nuova serie II: 181-342. Roma.

GóngorA, M. DE (1868): Antigüedades Prehistóricas de Andalucía. Imp. C. Moro. Madrid.

Mueller-BECK, H. (1965): "Seeberg, Burgäschisee-Süd, 5: Holzgeräte und Holzbearbeitung. Berne:Stämpfli”. Acta Bernensia, 21. Berne.

SCHLICHTHERLE, H. (1992): “Jungsteinzeitliche Erntegeräte am Bodensee". Plattform, 1/1992: 24-44. Unteruhldingen.

SCHWEINGRUBER, F.H. (1978): Mikroskopische holzanatomie. Zürcher A.G. Zug.

- (1990): Anatomie Europäischer Holzer.-Anatomy of European Woods. Eidgenössische Forschungsanstalt für Wald, Schnee und Landschaft, Birsmendorf (Hrsg). Haupt. Bern und Stuttgart

Tarrús, J.; Chinchilla, J.; Agustí, B.; Bosch, A.; BuXó, 
R.; Clop, X.; Faura, C.; NAvarro, C.; SAÑa, M. (1992): "La Draga: primer hábitat lacustre del Neolítico antiguo en el Mediterráneo occidental". Revista de Arqueología, 137: 8-18. Madrid.

- (1993): "Avanç sobre l'assentament lacustre de La Draga (Banyoles, Pla de l'Estany)”. Tribuna d'Arqueologia, 1991-92: 13-27. Barcelona.

TARRÚs, J.; ChINChilla, J.; BosCH, A. (1994)a: "La Draga (Banyoles): Un site lacustre du Néolithique ancien car- dial en Catalogne". Bulletin de la Société Préhistorique Française 91, 6: 449-456. Paris.

- (1994)b: "La Draga (Banyoles): un hàbitat lacustre vora l'Estany, ara fa 7.000 anys". Quaderns 1992 1994. Centre d'Estudis Comarcals de Banyoles: 57-79. Banyoles.

VorUZ, J.L. (1991): Le Néolithique suisse. Bilan documentaire. Document du Département d'Anthropologie et d'Ecologie de la Université de Genève, 16.

\section{GTAPI}

GRUPO DE TRABAJO DE ARQUEOBOTÁNICA DE LA PENÍNSULA IBÉRICA

Los días 27 y 28 de Enero de 1996 se ha llevado

a cabo la Primera Reunión del GTAPI en la Universidad

Autónoma de Barcelona, a la que asistieron los

siguientes investigadores:

D. Canal e I. González (Centre d'Investigacions Arqueològiques de Girona)

I. Peña-Chocarro (Universidad de Londres)

C. Cubero (CEM, Centre per a la Gestió per el patrimoni cultural i natural)

A. M. Arnanz (CEH, CSIC)

N. Rovira Buendía (Universidad Pompeu Fabra)

V. López (Botanisches Institut, Basilea)

L. Zapata (UPV, EUH)

G. Pérez Jordà (Universidad de Valencia)

N. Alonso (Universidad de Lleida)

Se tiene prevista la realización de reuniones periódica de trabajo en las que se contará con la participación de especialistas de reconocido prestigio internacional.

El Grupo de Trabajo está abierto a todo aquel interesado en esta disciplina.

\section{OBdETIVOS}

- Promover y consolidar los estudios arqueobotánicos en la Península Ibérica.

- Unificación de criterios metodológicos y su difusión entre la comunidad arqueológica.

Para contactar, dirigirse a la siguiente dirección:

Ana M. ${ }^{\mathrm{a}}$ Arnanz

Laboratorio de Arqueobotánica

Centro de Estudios Históricos, CSIC

$\mathrm{C} /$ Duque de Medinaceli, 8

28014 - Madrid (España) 